$\frac{3 y}{47^{1 / 36}}$ wis. (1)

(12)

Df. $1680-2$

DCID--20616

DE86 009619

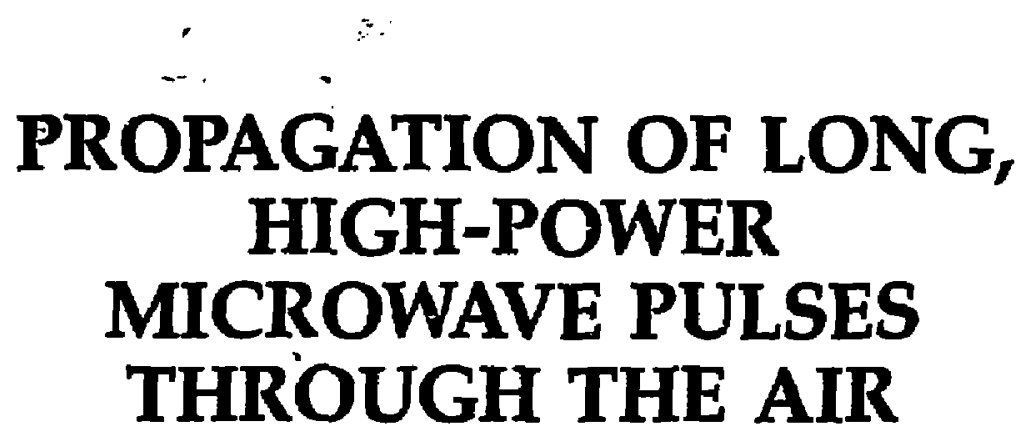

Gizzing H. Khanaka and Jick H. Yee

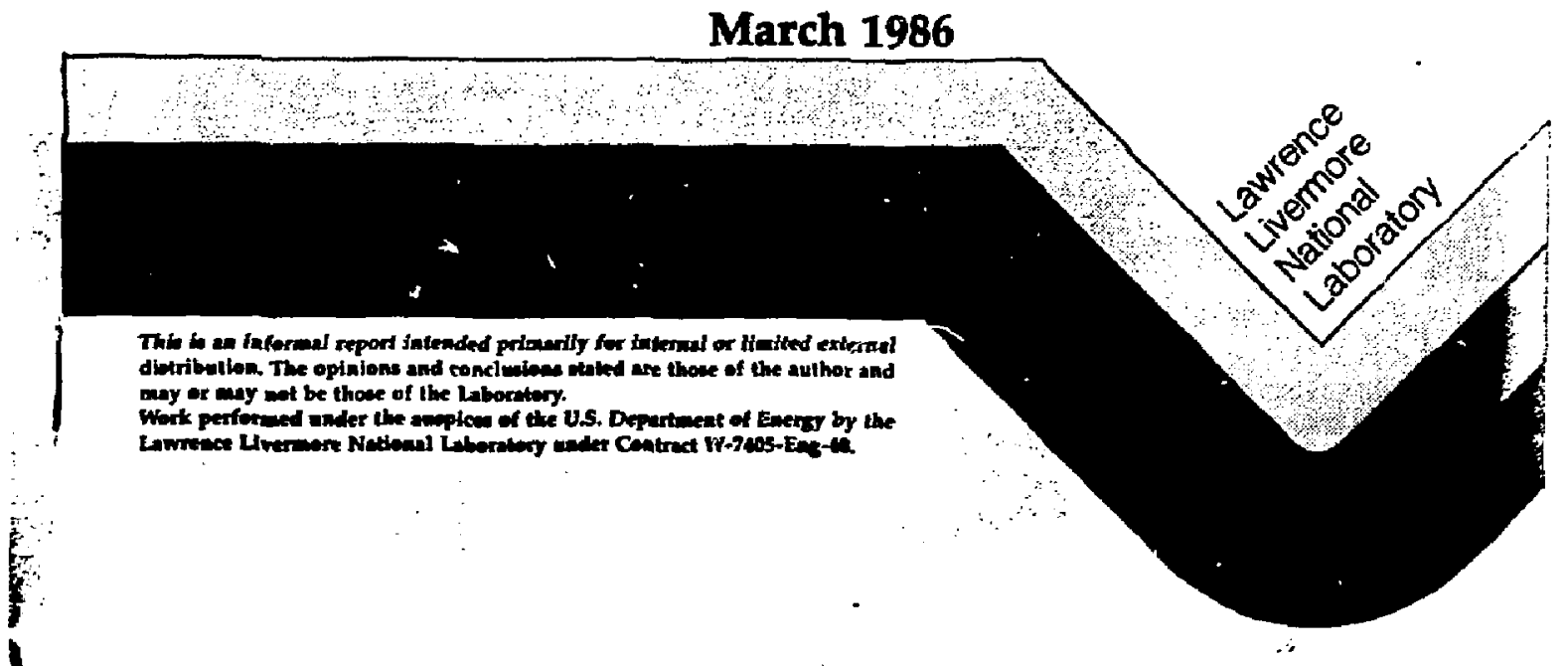

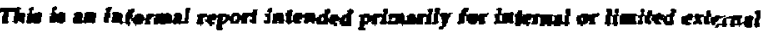
dietribulion. The opinions and conclwiess waled ave those of the author and may or way wet be those of the laboratery.

Work performed ander the anoptem of the U.S. Deppartment of Energy by the

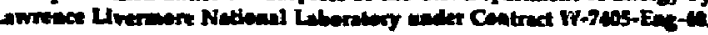

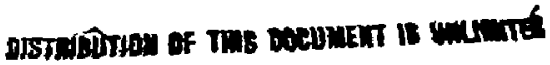


The propagation of high-power microwave pulses through the atmosphere has been a subject of investigation in the past few years. The primary objective of such work has been to assess the effect of microwave pulses on solid-state electronic systems, because a pulse of electromagnetic energy can induce destructive damages in such systems. Information on the energy and the : eak power that are transmitted through the atmosphere are needed in evaluating the threat posed by microwave pulses on communication equipment, radar, computers, etc. A one-dimensional computer code has been develop d to study the passage of a long, high-power microwave pulse at a constant altitude through the atmo here. In this note, the electron conductivity and density, as well as plasma frequency, particularly during : ? passage of the microwave pulse through the atmosphere, are addressed.

In the computer model, electrons are treated as a fluid, and their effect on the passing microwave pulse is determined by means of the simultaneous solution of Maxwell's equations and the ef tron fluid equations. The model should give fairly accurate predictions, because it takes into account suci: variables as ambient electron density, beam intensity, ionization (avalanche) and attachment rates, and atmospheric pressure.

An electromagnetic pulse of specific frequency, shape, intensity, and length is assumed to exist at some desired altitude. Figure 1 shows the pulse type used in this study. This pulse is then ass med to propagate through the atmosphere at a constant altitude for a specified distance. The computer -ode is designed to focus on the pulse region and monitor the activities of both the electrons and the elect smagnetic wave. In this code, all of the time intervals and distances are calculated relative to the front $7 d$ of the electromagnetic pulse. The code produces plots of the electric field amplitude and the electron $c$. רsity, energy, conductivity, and velocity at specified time intervals. It also calculates the average and maximum electron conductivity, the peak electron density, and the peak plasma frequency at each time interval. Later, time history plots are made for these parameters.

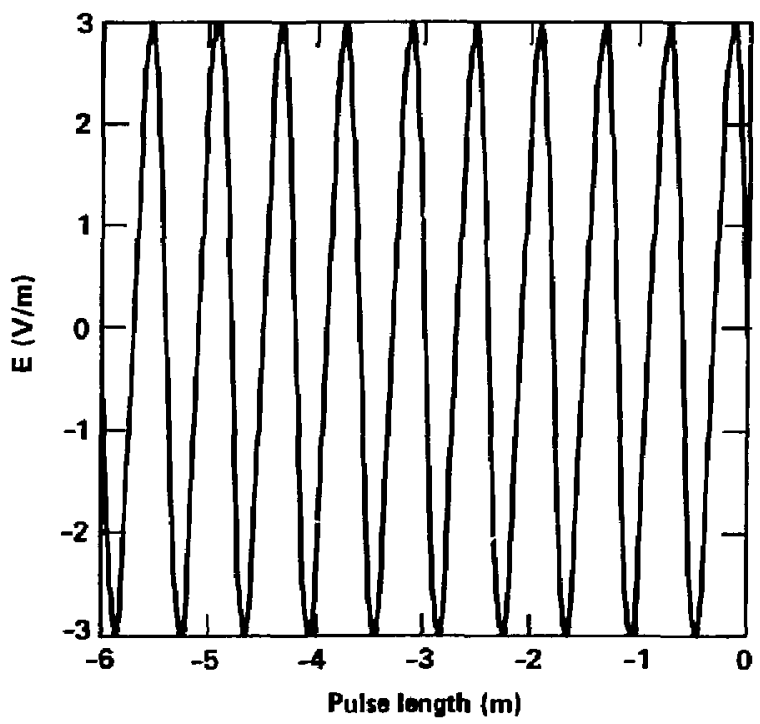

Figure 1. A sinusoidal pulse of $f=0.5 \mathrm{GHz}, T=2 \mathrm{~ns}$, $\mathrm{L}=0.6 \mathrm{~m}$, pulse length $=6 \mathrm{~m}$, pulse duration $=20 \mathrm{~ns}$. 


\section{The Electron-Fluid Model}

The electron-fluid model describes the passage of a plane microwave beam through the air by means of the following equations:

$$
\begin{aligned}
& \partial \mathrm{E} / \partial \mathrm{t}=-(\partial \mathrm{H} / \partial 2+\mathrm{nev}) / \varepsilon_{0} \\
& \partial \mathrm{H} / \partial \mathrm{t}=-(\partial \mathrm{E} / \partial z) / \mu_{0} \\
& \partial n / \partial t=n\left(v_{i}-v_{a}\right) \\
& \partial v / \partial t=e E / m-v\left(v_{\text {m }}+v_{i}-v_{2}\right) \\
& \partial u / \partial t=-2 E v / 3-u\left(v_{u}+v_{i}-v_{2}\right)+u_{0} v_{u}-2 E_{c} v_{l} / 3 \text {, }
\end{aligned}
$$

where $E$ is the electric field $(V / m)$ in the $x$ direction, and $H$ is the magnetic field $(A / m)$ in the $y$ direction. The wave propagates in the $z$ direction, and $e, m, n, v$, and $u$ are the electron charge $(C)$, mass (kg), density $\left(\right.$ elec $\left./ \mathrm{m}^{3}\right)$, velocity $(\mathrm{m} / \mathrm{s})$, and energy $(\mathrm{eV})$, respectively. The parameters $v_{j}, v_{n}, v_{m}$, and $v_{u}$ are the ionization $\left(s^{-1}\right)$, attachment $\left(s^{-1}\right)$, momentum transfer $\left(s^{-1}\right)$, and energy loss rates $\left(s^{-1}\right)$, respectively. $E_{c}$ is the ionization energy of air molecules (eV), and $u_{0}$ is the initial energy of air molecules $(e V) . \varepsilon_{0}$ and $\mu_{0}$ are the free-space permittivity $(\mathrm{F} / \mathrm{m})$ and permeability $(\mathrm{H} / \mathrm{m})$, respectively. Finally, $t$ is the time (s).

The electron conductivity and the plasma frequency are given by the following equations:

$$
\begin{aligned}
& g=n e^{2} / m v_{m} \\
& w_{p}=\left(n e^{2} / m \varepsilon_{0}\right)^{1 / 2}
\end{aligned}
$$

where $g$ is the electron conductivity (mho/m) and $w_{p}$ is the plasma frequency (rad/s).

The electron conductivity is a function of electron density and energy (via the momentum transfer rate). At low altitudes, the conductivity is more affected by the density than by the energy, because the electrons collide frequently with the air molecules. Hence, their energy does not change rapidly enough to cause significant change in the momentum transfer rate. At high altitudes, where the air is less dense, the electrons can gain significant amounts of energy from the electric field before a collision. Hence, the momentum transfer rate is expected to affect the conductivity data. The plasma frequency is a function of the electron density only; hence, its behavior is affected by the electron density. Equations (1) through (5) have to be solved simultaneously in a two-dimensional space $(z, t)$. It is clear that they form a set of coupled, nonlinear partial differential equations with no closed-form solution. In addition, the rates $v_{i}, v_{2}$, $v_{m}$ and $v_{u}$ are functions of the electron energy and atmospheric pressure. The functional dependency of these rates on the electron energy is shown in Fig. 2 Thus, numerical methods are employed to obtain an approximate solution for this problem.

\section{The Physics of Wave Propagation}

The results of this study can be understood by means of the physics of the problem. The propagation of electromagnetic waves in matter, especially in air, is accompanied by many physical processes that adversely affect the transportation of electromagnetic waves. Air breakdown is considered to be one of the important processes, because it causes attenuation. Thus, determining the breakdown threshold as a function of frequency and intersity is essential whenever information on the transportation of electromagnetic waves is desired. Hence, it is expected that the front portion of a high-power microwave pulse can pass through the air, while the back portion (tail) erodes. Figure 3 shows the dependency of the transmitted portion of the pulse on the intensity, frequency, and length of that pulse.

This type of behavior can be explained by examining the interaction between the electrons and the electromagnetic wave. As the pulse starts to propagate, the electric field accelerates the ambient free electrons. If the field is intense enough, the electrons gain enough energy to ionize air molecules and liberate more electrons. These electrons in turn are accelerated by the field and can cause further ionization in the atmosphere. At atmospheric pressure, the time development of the electron density is described by Eq. (3); it is obvious that the electron ionization rate must exceed the electron attachment rate for air breakdown to occur. The threshold intensity for air breakdown is defined by the intensity at which 

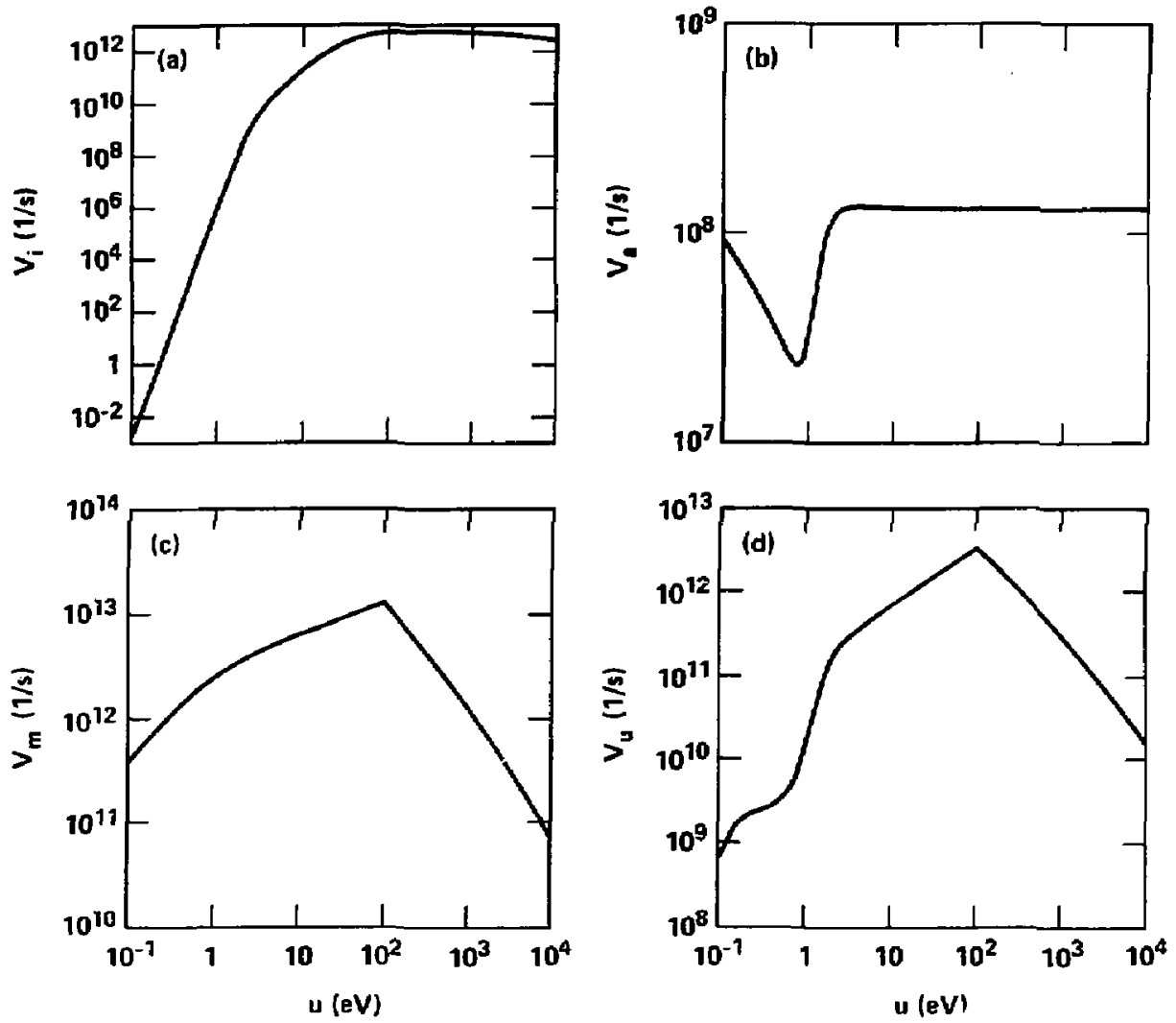

Figure 2. Ionization $\left(v_{p}\right)$, atlachment $\left(v_{-:}\right.$- momentum transfer $\left(v_{m}\right)$, and energy loss $\left(v_{0}\right)$ rates as a function of electron energy at sea level.

tail erosion is initiated. Figure 4 shows the time development of the electron density for a pulse intensity higher than the threshold intensity; the electron density in this case increased rapidly in a short time interval. For intensities above the threshold, the electron density usually reachs a high value $\left(10^{14}-10^{19}\right.$ elec $/ \mathrm{m}^{3}$ ) in nanoseconds. This study shows that tail erosion does not occur as long as the density is below $10^{14} \mathrm{elec} / \mathrm{m}^{3}$. Once the tail of the pulse has eroded significantly, then the electron density starts to decrease. The manner and the speed by which the density increases and then decreases depends on the frequency and intensity of the pulse.

In summary, the front portion of an intense electromagnetic pulse creates an ionized atmosphere through which the tail has to pass. The creation of an ionized medium in the air has several interesting effects. Of particular interest here is the fact that the ionized air acts as an attenuator or a reflector for the incoming electromagnetic wave. Therefore, when the tail portion of the pulse passes through such an atmosphere, it is subject to attenuation and possibly reflection. Hence, it loses a significant amount of its energy to the electrons. The energy absorbed by these electrons is used in tonizing, exciting, and heating up the molecules. 

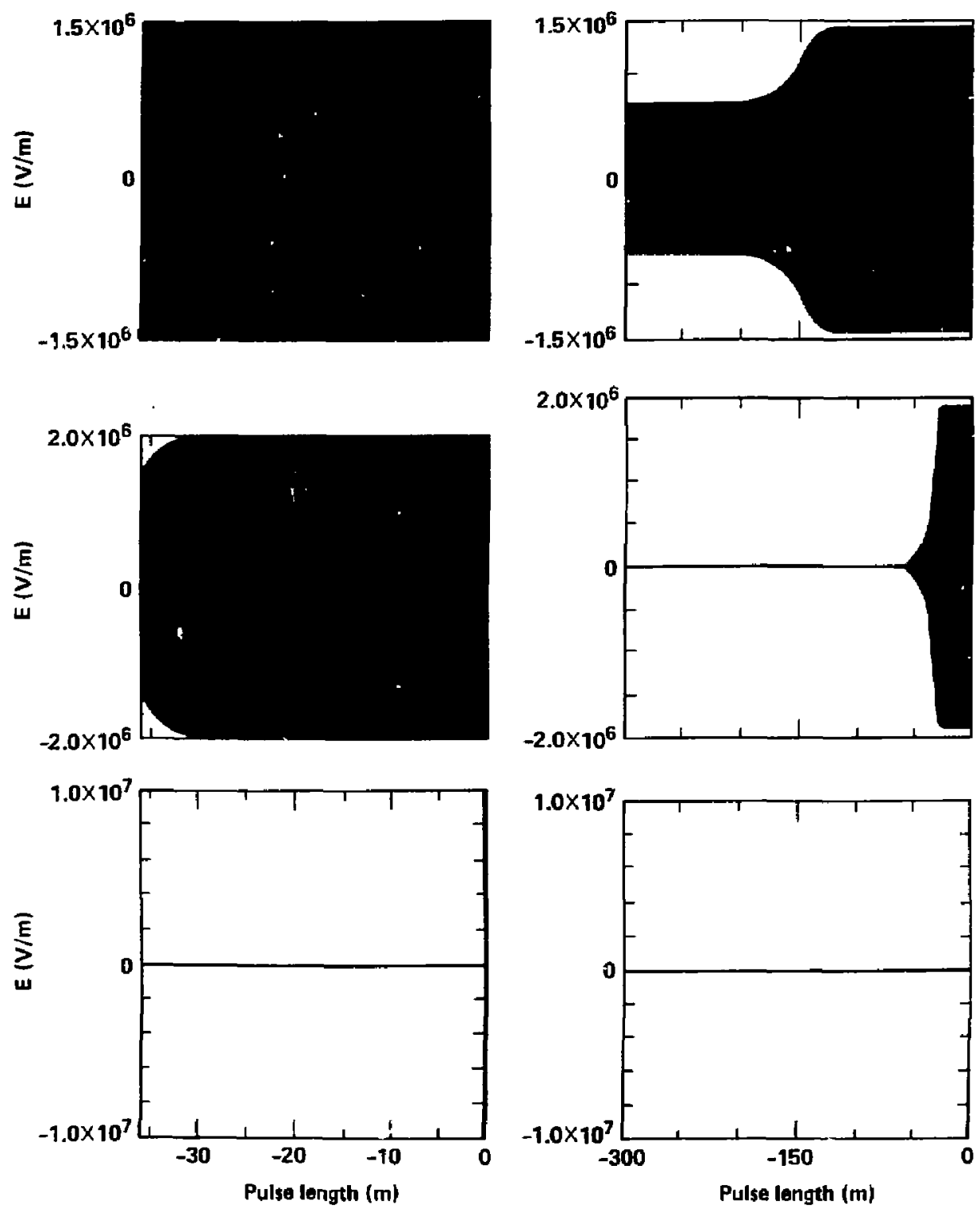

(a) $f=5 \mathrm{GHz}$

(b) $f=0.5 \mathrm{BHz}$

Figzre 3. Plots of the electric field amplitude vs pulse length showing the dependency of the transmitted portion of a microwave pulse on intensity, frequency, and length of the pulse. These Fot represent the electric field amplitudes at (a) $t=1 \mu \mathrm{s}$ and (b) $t=1.5 \mu \mathrm{s}$. 

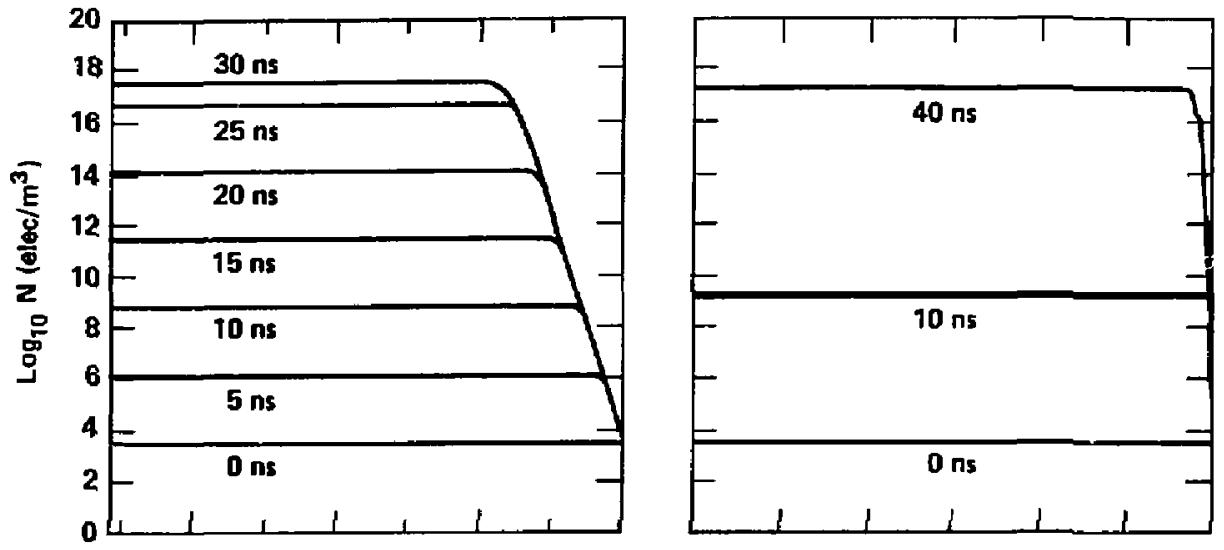

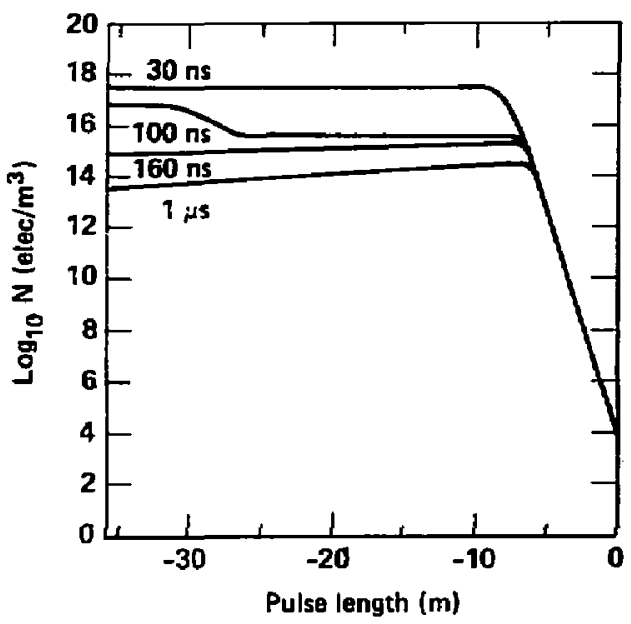

(a) $f=\mathbf{5} \mathbf{~ G H z}$

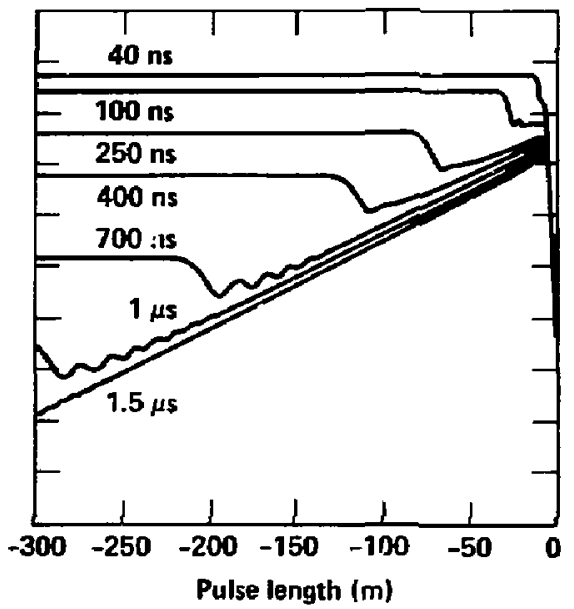

(b) $f=0.5 \mathrm{GHz}$

Figure 4. Plots showing the time development of the electron density for a field amplitude of 3 $\mathrm{MV} / \mathrm{m}$, where the electron density increases with time until it reaches a maximum value. It takes 30 ns i $\mathrm{i}$, the 5-GHz pulse and 40 ns for the $0.5-\mathrm{GHz}$ pulse to reach the maximum value. At this point, tail erosion is initialed; its effect on the electron density is seen in the lower graphs.

\section{Electron Conductivity}

The electron conductivity was studied for two pulses: a 5-GHz pulse of $120-\mathrm{ns}$ duration ( $36 \mathrm{~m}$ long) and a $0.5-\mathrm{GHz}$ pulse of $1-\mu \mathrm{s}$ duration ( $300 \mathrm{~m}$ long). In both cases, the altitude was kept constant at $6.1 \mathrm{~km}$ $\left(20,000 \mathrm{ft}\right.$ ), and the electric field amplitude was varied from $1-10 \mathrm{MV} / \mathrm{m}$ (i.e., an intensity of $1.33 \times 10^{9}$ $1.33 \times 10^{11} \mathrm{~W} / \mathrm{m}^{2}$ ). The pulses were allowed to travel a distance longer than their lengths $(300 \mathrm{~m}$ for the 5$\mathrm{GHz}$ pulse and $450 \mathrm{~m}$ for the $0.5-\mathrm{GHz}$ pulse). 
This study shows that there is a specific air breakdown threshold associated with each pulse. The obtained threshold level is $1.4 \mathrm{MV} / \mathrm{m}$ for the $0.5-\mathrm{GHz}$ pulse and $2.0 \mathrm{MV} / \mathrm{m}$ for the $5-\mathrm{GHz}$ pulse. The electron conductivity is affected greatly by the term $\left(v_{i}-v_{j}\right)$ in Eq. (3). For pulse amplindes below the threshold level, the term $\left(v_{i}-v_{a}\right)$ increases or decreases slowly with time; hence, the electron conductivity increases or decreases very moderately as in Fig. 5 . When the intensity exceeds the threshold level, the electron conductivity increases by orders of magnitude over a short period of time, as shown in Fig. 6 . This behavior in the electron conductivity is attributed to electron avalanche.

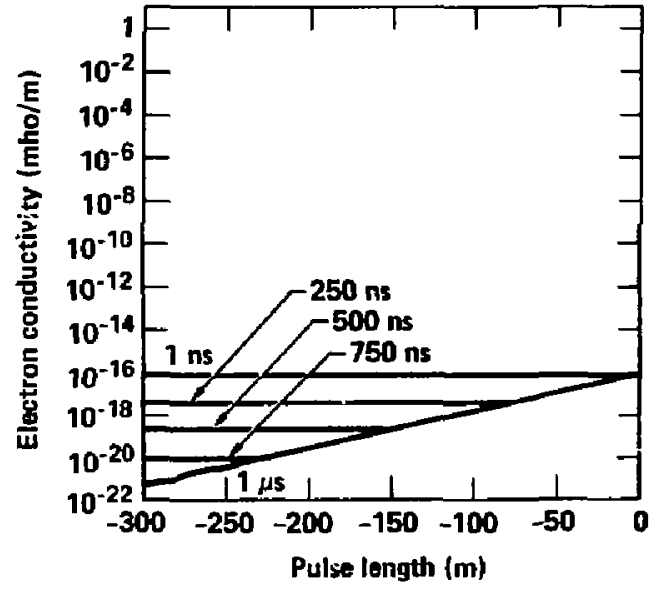

(a) $\mathrm{A}=1 \mathrm{MV} / \mathrm{m}$

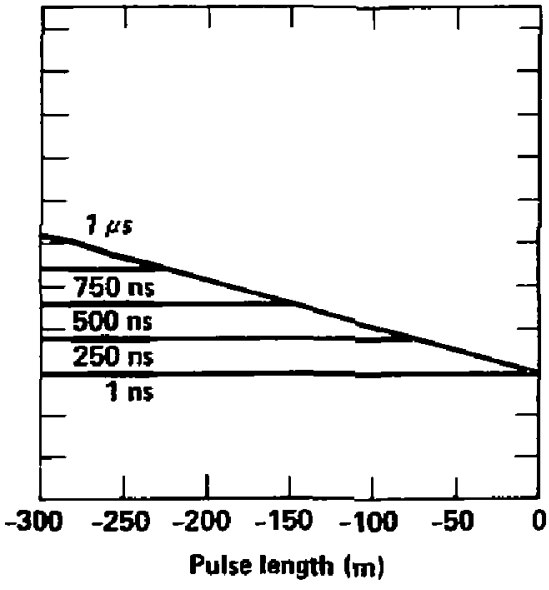

(b) $A=1.3 \mathrm{MV} / \mathrm{m}$

Figure 5. Plots demonstrating the time development of the electron conductivity for the 0.5-GHz pulse with field ampiitudes below the avalanche ionization intensity.
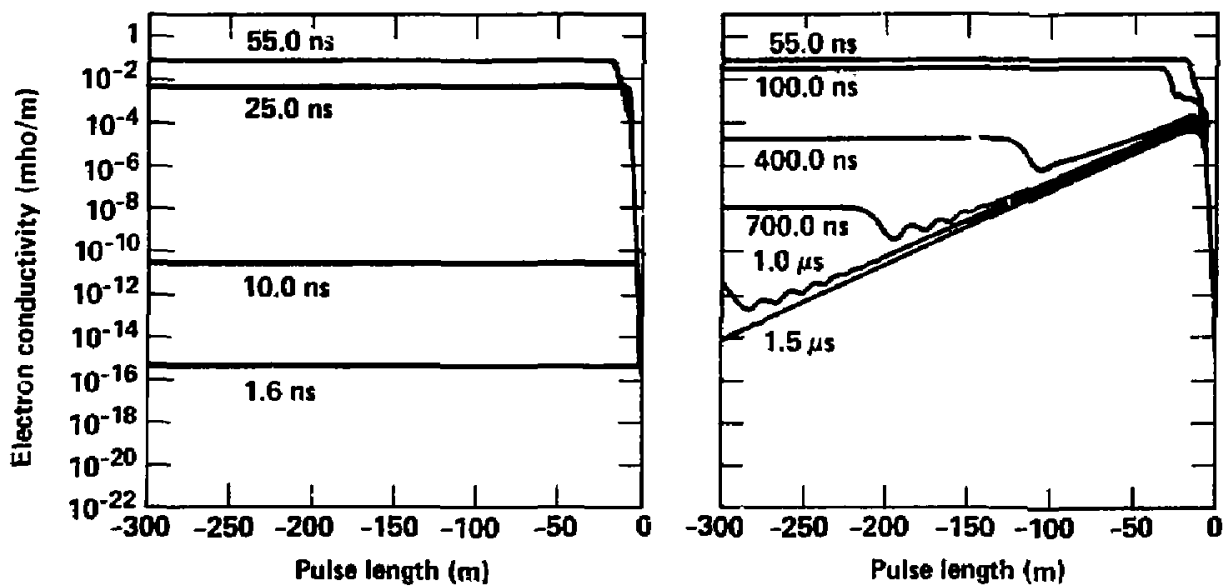

Figure 6. Conductivity values for pulse amplitude of $3 \mathrm{Mv} / \mathrm{m}$, which is above the threshold level. The conductivity reaches its peak value in $55 \mathrm{~ns}$. Then, it starts to decrease gradually. 
At the end of each time interval, the average and maximum electron conductivity and the peak electron density were obtained. The peak plasma frequency was calculated by using the peak eiection density in Eq. (7). All of these data points were used to produce Figs. 7-10. It is clear from these figures that the general behavior of these parameters is very similar for both pulses. The majot difference is in their threshold level.

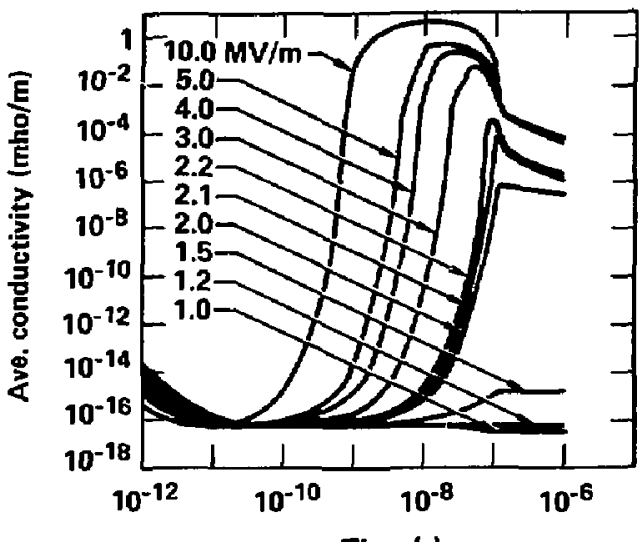

Time (s)

(a) $f=5 \mathrm{GHz}$

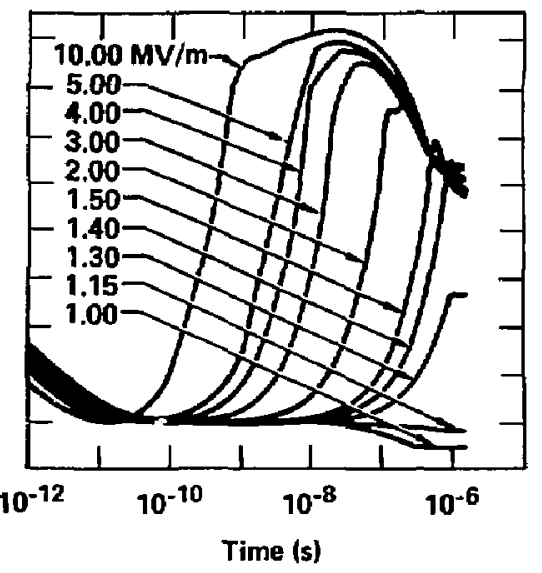

(b) $f=0.5 \mathrm{GHz}$

Figure 7. Plots of the average electron conductivity vs time for field amplitudes ranging from 1-10 $\mathrm{MV} / \mathrm{m}$.

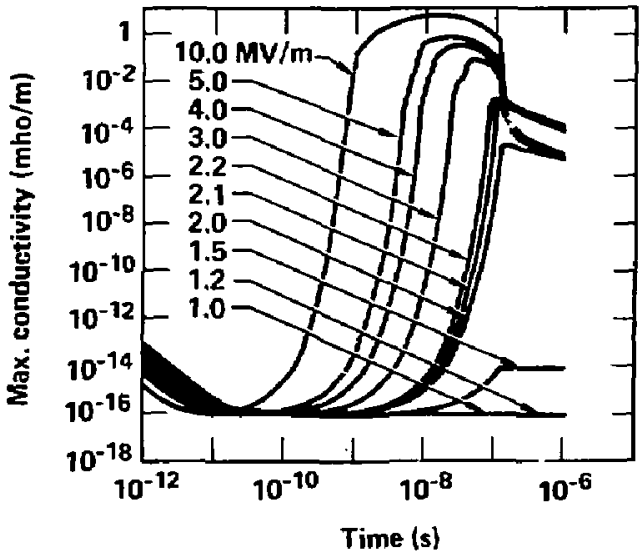

(a) $f=5 \mathrm{GHz}$

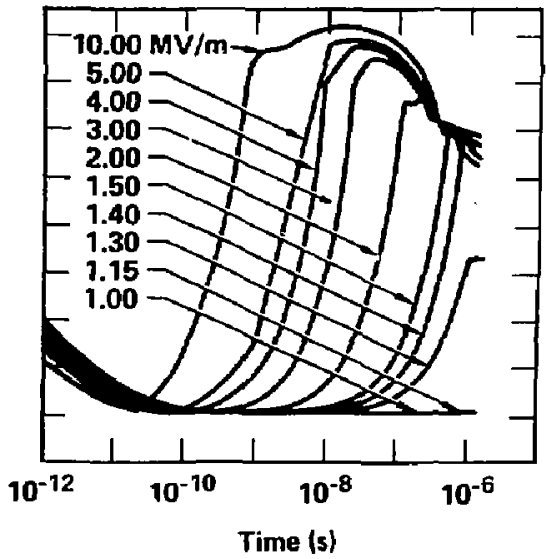

(b) $f=0.5 \mathrm{GHz}$

Figure 8. The maximum electron conductivity plotfed as a function of time with field amplitudes of 1-10 MV/m for both pulses. 


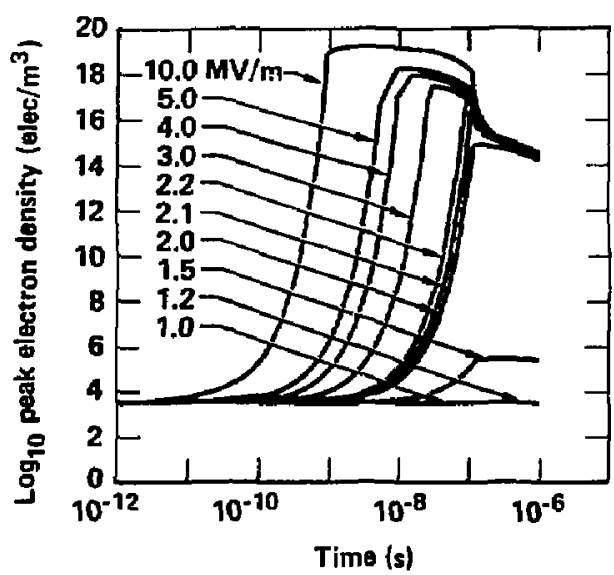

(a) $\mathbf{f}=\mathbf{5} \mathbf{~ G H z}$

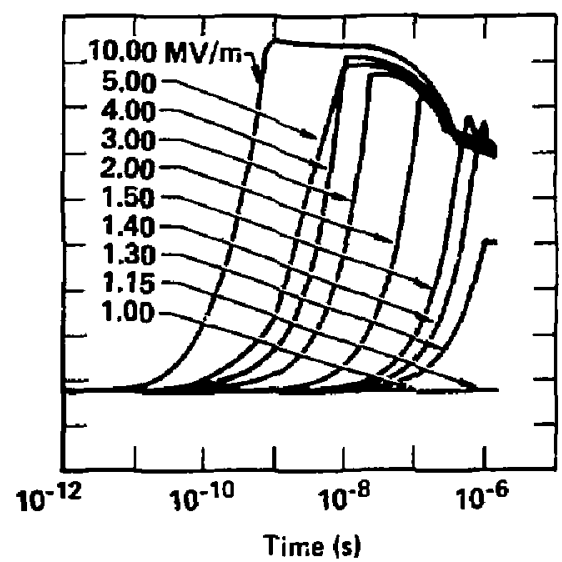

(b) $f=0.5 \mathrm{GHz}$

Figure 9. Plots of the peak electron density as a function of time for both pulses. The electric field amplitude varies from $1-10 \mathrm{MV} / \mathrm{m}$.

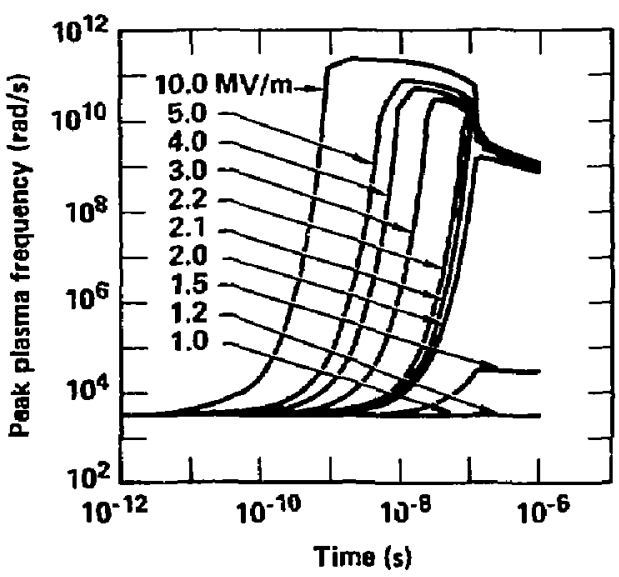

(a) $\mathbf{f}=\mathbf{5} \mathrm{GHz}$

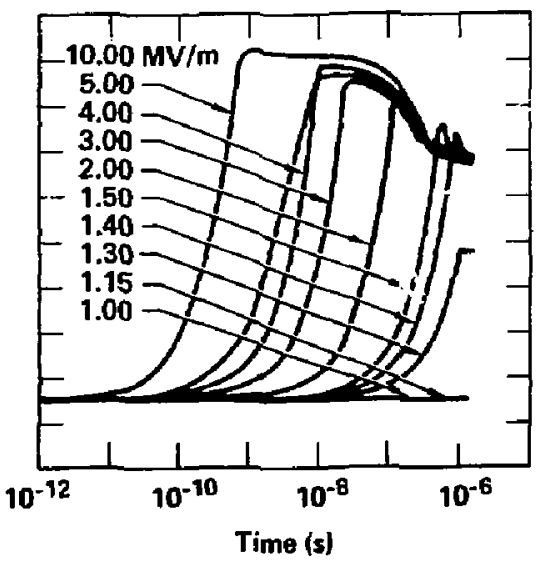

(b) $f=0.5 \mathrm{GHz}$

Figure 10. Plots of the peak plasma frequency as a fanction of time for both pulses. The electric field amplitude varies from $1-10 \mathrm{MV} / \mathrm{m}$.

Figure 10 shows the dependency of the peak plasma frequency on time for both cases. It shows that the plasma frequency exceeds $5 \mathrm{GHz}$ when the field amplitude is equal to or greater than $4 \mathrm{MV} / \mathrm{m}$. This indicates that the ionized atnosphere is acting as a reflector for the inconing microwave pulse. Similarly, the plasma frequency exceeds $0.5 \mathrm{GHz}$ when the field amplitude is equal to or greater than $1.5 \mathrm{MV} / \mathrm{m}$. 


\section{Summary}

The passage of long, high-power microwave pulses in the atmosphere was studied using a onedimensional computer code. The objective of this study was to obtain a time history of the electron conductivity and peak density, as well as the peak plasma density. The results are summarized as follows:

- The threshold level depends on pulse frequency and length.

- Electron avalanche occurs only when the pulse intensity exceeds the threshold level for air breakdown, and this results in tail erosion.

- For higher pulse intensities, it requires less time to initiate electron avalanche, which results in transmitting smaller portions of the pulse.

- The general characteristics of the electron density, conductivity, and plasma frequency are quite similar for both cases. rise.

- As the pulse amplitude rises, the electron conductivity and density and the plasma frequency also 


\section{Contents}

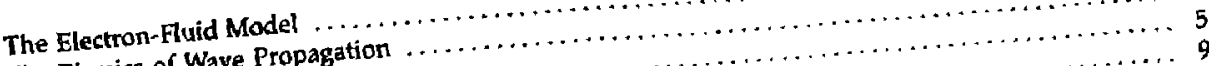

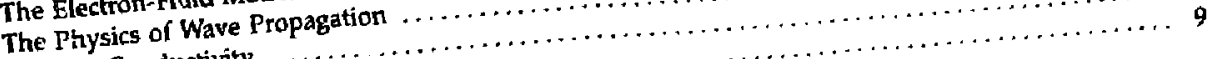

Electron Conductivity

Summary

\section{DISCLAIMER}

This report was prepayed as an account of work sponsored by an agency of the United States Government. Neither the United States Government nor any agency thereof, nor any of their employees, makes any warranty, express or implied, or assumes any legal liability or responsibitity for the accuracy, completenss, or usefulness of any information, apparatus, product, or process discloced, of fepresents that its use would not infrioge privately owned rights. Reference herein to any specific commencial product, process, or service by irade aame, trademark, manufacturer, or aberwise does not aecessarily constiute or imply its endorsement, recommeadation, or faworiag by the United Siates Goveroment or any agency thereof. The views and opinions of authors expresced herein do not neossarily state or refloct those of the United States Government on any a gency thereef. 